\title{
Stability of Magneto-optical Traps with Large Field Gradients: Limits on the Tight Confinement of Single Atoms
}

\author{
P. A. Willems, * R. A. Boyd, J. L. Bliss, and K. G. Libbrecht ${ }^{\dagger}$ \\ Norman Bridge Laboratory of Physics, 264-33 California Institute of Physics, Pasadena, California 91125
}

(Received 5 August 1996)

\begin{abstract}
We report measurements of the stability of magneto-optical traps (MOTs) for neutral atoms in the limit of tight confinement of a single atom. For quadrupole magnetic field gradients at the trap center greater than $\sim 1 \mathrm{kG} / \mathrm{cm}$, we find that stochastic diffusion of atoms out of the trapping volume becomes the dominant particle loss mechanism, ultimately limiting the MOT size to greater than $\sim 5 \mu \mathrm{m}$. We measured and modeled the diffusive loss rate as a function of laser power, detuning, and field gradient for trapped cesium atoms. In addition, for as few as two atoms, the collisional loss rates become very high for tightly confined traps, allowing the direct observation of isolated two-body atomic collisions in a MOT. [S0031-9007(97)02514-3]

PACS numbers: $32.80 . P \mathrm{j}, 39.10 .+\mathrm{j}$
\end{abstract}

The magneto-optical trap (MOT) [1] is a useful tool for producing large numbers of laser-cooled and trapped neutral atoms at sub-mK temperatures. Many recent experimental investigations of ultracold atomic collisions, atom optics and interferometry, and gaseous Bose-Einstein condensation have used MOTs as a first step in the preparation of cold atom samples. The MOT itself has been the focus of numerous studies, which have examined collective behavior from radiation trapping [2], collisional losses [3], and polarization-gradient cooling [4,5]. In this Letter we examine the potential for using MOTs to confine neutral atoms to small and well-defined regions of space. In this "tight confinement" limit, one makes a connection to investigations of quantum-limited atomic position measurements in optical fields [6], and, in addition, addresses the physical limits of magneto-optical lenses for focusing atomic beams [7]. The tightest possible confinement of small numbers of magneto-optically trapped radioactive atoms may also be desirable [8].

Because polarization-gradient cooling is quite effective in MOTs [4,5], a significant reduction in trap size via improved cooling would require cooling below the singlephoton recoil limit, which has not yet been realized in MOTs. The alternative for achieving tighter atom confinement is to increase the trap spring constant, which is expected to be proportional to magnetic field gradient [1]. We have found, using measurements of the dynamical behavior of trapped atoms, that for typical MOT parameters the spring constant is indeed linearly dependent on the quadrupole magnetic field gradient, up to axial gradients of at least $B^{\prime}=d B_{z} / d z=1.4 \mathrm{kG} / \mathrm{cm}$, while the damping constant remains independent of $B^{\prime}$ [9]. Thus the trap size is expected to be proportional to $\left(B^{\prime}\right)^{-1 / 2}$. We identify here a new diffusive loss mechanism that ultimately limits the trap stability at high $B^{\prime}$, and thus limits the degree to which neutral atoms can be stably confined in a MOT.

The stability of a MOT is determined by the different mechanisms that remove atoms from the trap. These can be parametrized by a trap population rate equation,

$$
d N / d t=R-\left(\tau_{\text {back }}^{-1}+\tau_{\text {diff }}^{-1}\right) N-\beta N(N-1),
$$

where $N$ is the number of atoms in the trap, $R$ is the MOT loading rate, $\tau_{\text {back }}$ is the trap lifetime due to background collisions, $\beta$ arises from collisions between trapped atoms [10], and $\tau_{\text {diff }}$ is the mean time for diffusion of atoms out of the trapping volume. Each of the parameters in turn depends on the characteristics of the MOT, such as the laser intensity, detuning from the atomic resonance, beam size, and the quadrupole field gradient. The parameters also depend on $N$ when $N$ is large, owing to collective effects between atoms [2].

Our experimental investigations of tightly confined MOTs were performed in a vacuum chamber cooled to below $4 \mathrm{~K}$, inside of which MOTs were formed [11]. With this system we could produce high quadrupole field gradients using superconducting magnetic field coils, while background collisional losses gave $\tau_{\text {back }} \approx 1 \mathrm{~h}$. A copper shutter at $4 \mathrm{~K}$ in the side of the chamber admitted a $450 \mathrm{~K}$ thermal cesium beam to load atoms into the MOTs. An intensified charge-coupled device (CCD) camera provided sufficient sensitivity so that fluorescence from single atoms could be observed on a live video image [12].

Figure 1 shows a measurement of the average residence time $\langle\tau\rangle$ for atoms in a MOT, as a function of $B^{\prime}$, for a single laser intensity and detuning. This was determined by forming a stable MOT and then measuring the average time before the atom(s) escaped. For trap populations as small as $N=2$, collisional losses dominate over other loss mechanisms as $B^{\prime}$ is increased; these losses are consistent with the typical collisional loss rates in MOTs [3]. Furthermore, we found that a high-field-gradient MOT with two atoms almost always decayed to an empty MOT, which we interpret as the direct observation of isolated two-body collisions between trapped atoms.

For single-atom MOTs the trap loss at high $B^{\prime}$ was dominated by the stochastic diffusion of the atom out of the 


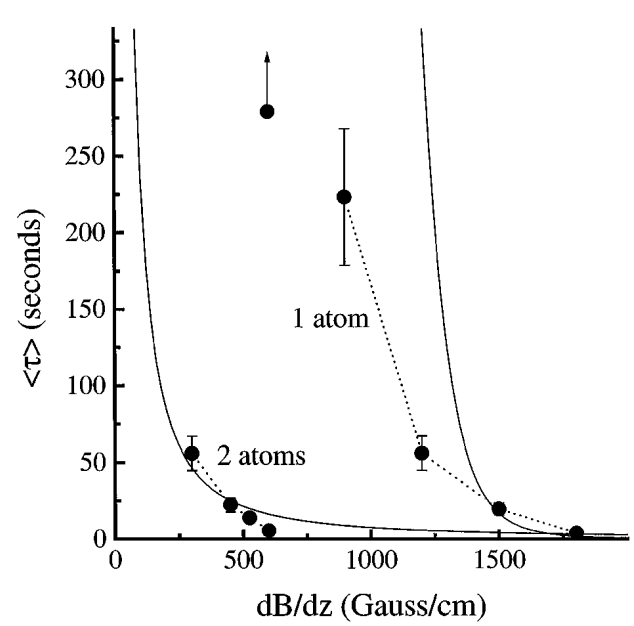

FIG. 1. Average residence time $\langle\tau\rangle$ for one and two cesium atoms in a MOT, as a function of the axial magnetic field gradient, for fixed detuning $\delta / \Gamma=1.35$ and single-beam laser intensity $I / I_{\text {sat }}=16$. Two-atom MOTs decay via collisions between the two atoms, while single atoms decay via diffusion out of the trapping volume. The curve through the twoatom points shows the expected decay time using a twobody collision rate $\beta^{*}=2 \times 10^{-11} \mathrm{~cm}^{3} / \mathrm{sec}$ [13]. The curve through the one-atom points is from the phenomenological diffusion model described in the text, with $\eta=3.6$.

MOT capture volume. To examine the behavior of the diffusive loss-rate parameter $\tau_{\text {diff }}$ as a function of laser intensity and detuning, we first loaded several atoms into the MOT, increased $B^{\prime}$ until one atom remained, then continued to increase $B^{\prime}$ until the atom was observed to leave the trap, with $d B^{\prime} / d t$ such to give $\langle\tau\rangle \approx \tau_{\text {diff }} \approx 1 \mathrm{sec}$. This procedure was repeated several times to check reproducibility, giving a measure of the field gradient $B^{\prime}$ for which $\tau_{\text {diff }} \approx 1 \mathrm{sec}$. Figure 2 shows these measurements over a range of laser intensities and detunings.

To model the single-atom MOT data in Figs. 1 and 2 we calculate the behavior of the diffusive loss coefficient $\tau_{\text {diff }}$ using a 1D Fokker-Planck diffusion model. We first make the simplifying assumption that the atomic motion in the MOT is everywhere strongly overdamped. Dynamic studies of MOTs have shown that this assumption is justified, at least near the trap center, even for large laser intensities and large $B^{\prime}$, due to the effects of polarization-gradient cooling $[9,14]$. With this assumption the Fokker-Planck equation reduces to the Smoluchowski equation [15], and the rate for the diffusion of atoms out of the trap is given by

$$
\tau_{\mathrm{diff}}^{-1}=\left(\frac{\kappa k_{B} T}{2 \pi \alpha^{2}}\right)^{1 / 2} / \int_{0}^{b} e^{U(z) / k_{B} T} d z
$$

where $\alpha$ is the usual MOT damping coefficient (defined by the damping force $F=-\alpha v$, where $v$ is the atom velocity), $\kappa$ is the spring constant at the trap center $(F=-\kappa z), T$ is the trap temperature, and $U(z)$ is the MOT pseudopotential ( $U \approx \frac{1}{2} \kappa z^{2}$ near the trap origin). The $1 \mathrm{D}$ equation suffices for this because the $3 \mathrm{D}$ equation

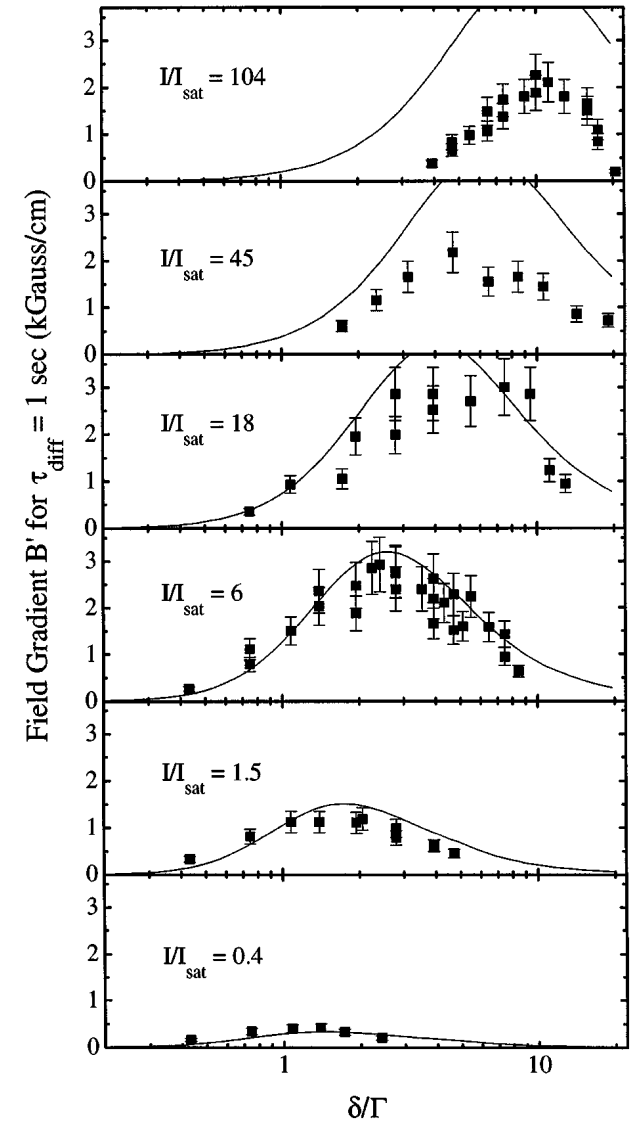

FIG. 2. Measured MOT axial field gradient $B^{\prime}$ at which the diffusion parameter is $\tau_{\text {diff }}=1 \mathrm{sec}$, as a function of laser detuning $\delta$, for several different laser intensities. The smooth curves are from the phenomenological diffusion model described in the text, with $\eta=3$.

separates, and in Monte Carlo simulations we find that atoms always diffuse out of the trap along the axial direction (which is the most tightly confining). The integral is from $z=0$, the trap center, to $z=b$, the point at which atoms are no longer confined by the trap. We can approximate the integrand by $U(z) \approx-\kappa b^{2} / 2+$ $\kappa b z$, which is an expansion about $z=b$, giving the analytic expression

$$
\tau_{\mathrm{diff}}^{-1} \approx \frac{1}{\sqrt{\pi}} \frac{\kappa}{\alpha}\left(\frac{\kappa b^{2}}{2 k_{B} T}\right)^{1 / 2} e^{-\kappa b^{2} / 2 k_{B} T} .
$$

Evaluation of this expression requires knowledge of two quantities, the MOT relaxation time $\tau_{\text {relax }} \equiv \alpha / \kappa$ and the ratio of the overall trap depth $U_{0} \approx \frac{1}{2} \kappa b^{2}$ to the trap temperature $T$. To model our data these must be given as a function of laser intensity $I$ and detuning $\delta$, and as a function of field gradient $B^{\prime}$. Our theoretical understanding of these parameters is unfortunately incomplete due in part to complications of sub-Doppler cooling mechanisms present for atoms possessing several nearly degenerate magnetic sublevels, and generalization of 1D theories to 3D MOTs. Theory is particularly uncertain at high laser 
intensity $I / I_{\text {sat }} \gg 1$, which applies for much of the data in Fig. 2, and for nonzero magnetic fields, such as are present at the edge of a MOT.

In spite of these theoretical uncertainties, we can nevertheless make crude analytical estimates of the parameters in the above expression for $\tau_{\text {diff }}^{-1}$, guided by recent measurements of MOT dynamics. The purpose of this is twofold, to test that the lifetime data in Fig. 2 are indeed consistent with our underlying hypothesis of diffusive loss and to model the minimum attainable MOT size. The latter is relevant in regard to magneto-optical focusing of atomic beams.

The MOT relaxation time $\tau_{\text {relax }}$ can be readily measured through observations of the motion of atoms trapped in a MOT, for example, by displacing the trap and observing its relaxation to equilibrium. Such measurements give values of $\tau_{\text {relax }}$ that are about $10 \times$ larger than predicted by 1D sub-Doppler theory, and show an unexpected linear dependence on detuning $\delta$ [5]. Our own dynamical measurements using cesium [9] give $\tau_{\text {relax }} /(1 \mathrm{sec}) \approx(0.03 \mathrm{G} / \mathrm{cm})\left(2 \delta / \Gamma B^{\prime}\right)$, where $\Gamma$ is the natural linewidth of the transition, independent of intensity for $1<I / I_{\mathrm{sat}}<15$ (for the cesium $S_{1 / 2} \rightarrow P_{3 / 2}$ transition, $\Gamma=2 \pi \times 5.2 \mathrm{MHz}$ and $I_{\text {sat }}=1.1 \mathrm{~mW} / \mathrm{cm}^{2}$ ).

The relative trap depth $U_{0} / k_{B} T$ is the most difficult parameter in the above expression to model theoretically, since $U_{0}$ depends crucially on the behavior of the MOT forces near the edge of the trap, where the Zeeman splitting is of the same order as the light shift of the ground state [16]. The transition from the low-field region near the trap center, where polarization-gradient forces are in effect, to the strong-field region is particularly problematic. Measurements of typical values of $U_{0}$ have been made using a pulsed disturbance [1] and using catalyzed cold collisions [17], but a systematic study measuring $U_{0}$ as a function of $I, \delta$, and $B^{\prime}$ has not yet been performed. Taking $U_{0}=\frac{1}{2} \kappa b^{2}$, however, we can proceed using phenomenological estimates of $T, \kappa$, and $b$ separately.

For low atom numbers, measurements of atom temperatures in MOTs are found to be identical with those measured in 3D $\sigma^{+}-\sigma^{-}$optical molasses, which for low intensities and large detunings $(\delta / \Gamma>5)$ is given by $T \approx$ $\left[2.6+60(\Gamma / 2 \delta)\left(I / I_{\mathrm{sat}}\right)\right] \mu \mathrm{K}$ for cesium [5]. At smaller detunings the temperature is larger than that given by this expression, in agreement with 3D calculations [18]; in this region $(\delta / \Gamma<5)$ the extrapolation $T \approx\left[260(\Gamma / 2 \delta)^{2}+\right.$ $\left.60(\Gamma / 2 \delta)\left(I / I_{\text {sat }}\right)\right] \mu \mathrm{K}$ describes the temperature data adequately for our model.

The spring constant can be calculated using 1D sub-Doppler theory, giving $\kappa \approx A \mu_{B} B^{\prime} k(2 \delta / \Gamma) /[1+$ $\left.(2 \delta / \Gamma)^{2}\right][2,4,19]$, where $A$ is a constant depending on atomic parameters. A number of measurements of the spring constant $\kappa$ have been made [4,5,9,19], with the result that the $1 \mathrm{D}$ theory gives roughly the correct functional form (over the limited intensity range approximately $0.4<I / I_{\mathrm{sat}}<4$ ), but overestimates the magnitude of $\kappa$ by about an order of magnitude. For cesium we find empirically that $A \approx 0.10 \pm 0.03$.

Semiclassical and quantum calculations have shown for large detuning that the boundary between the central and outer regions of a (two-component) MOT occurs at the radius $b \approx \eta\left(I / I_{\mathrm{sat}}\right)\left(\hbar \Gamma / \mu B^{\prime}\right)(\Gamma / 2 \delta)$, where $\eta$ is a numerical factor near unity [16]. At this radius the light shift of the atomic ground state equals the Zeeman shift, and the MOT restoring force reaches a local maximum. Beyond this radius the restoring force falls rapidly, and the effective temperature increases due to the suppression of sub-Doppler cooling mechanisms. Thus for a very tightly confined MOT we expect rapid diffusion of atoms out of the MOT beyond this radius.

Evaluating $\tau_{\text {diff }}^{-1}$, using the above estimates for $\tau_{\text {relax }}$, $T, \kappa$, and $b$, gives an adequate fit to our data at low intensities, where sub-Doppler theory and the MOT dynamical data are reasonably secure, but grossly overestimates the trap stability $\left(\tau_{\text {diff,calc }} \gg \tau_{\text {diff,observed }}\right)$ at higher intensities. This discrepancy is not surprising, since the sub-Doppler theory is known to break down at high laser intensities. We find, however, that if we add an ad hoc high-intensity rolloff to $U_{0}$, for example, taking $b=\eta\left(I / I_{\text {sat }}\right)\left(\hbar \Gamma / \mu B^{\prime}\right) \times$ $(2 \delta / \Gamma) /\left[1+6 I / I_{\mathrm{sat}}+(2 \delta / \Gamma)^{2}\right]$ (which equals the above expression for $b$ in the limit of low intensity and large detuning), we obtain a fairly good fit to the data, shown in Fig. 2. Note the prefactor $\eta$ serves as a scaling factor in Fig. 2, and is adjusted to fit the low-intensity data. Additional measurements of $U_{0}$ and $\kappa$ at high laser intensities, or a more reliable theoretical model of MOTs in this region, are necessary to quantitatively reproduce our MOT stability data at the higher intensities.

The above phenomenological model can be used to estimate the minimum attainable MOT size. This question is of particular importance in regard to the ultimate physical limits in using 2D MOTs for the tight focusing of neutral atomic beams. The smallest possible single-atom MOT can be formed by ramping up $B^{\prime}$ quickly, realizing a transient tight confinement before the atom diffuses away. The trap's finite response time gives the smallest MOT when $\tau_{\text {diff }} \approx \tau_{\text {relax }}$ or, equivalently, $\kappa b^{2} / k_{B} T \approx 1$. Taking the trap radius as $\kappa z^{2} \approx k_{B} T$, the minimum size is given by $z_{\min }(\delta, I) \approx k_{B} T / \kappa_{0} b_{0}$, where $\kappa_{0}=\kappa / B^{\prime}$ and $b_{0}=b B^{\prime}$. Assuming the same parameters used above to compute $\tau_{\text {diff }}^{-1}$, we find that $z_{\text {min }}(\delta, I)$ exhibits a broad minimum around $\delta / \Gamma \approx 2.5, I / I_{\mathrm{sat}} \approx 3$, with a global minimum MOT size of $z_{\text {global min }} \approx 2.4 \mu \mathrm{m}$. Note that the value of $z_{\text {global min }}$ depends only weakly on the details of our model; $z_{\text {global min }}$ is attained at moderate laser intensities, where the model is fairly secure, and is not sensitive to the form of high-intensity rolloff chosen. These results suggest that plain MOTs will not provide tight enough confinement to exhibit interesting fluorescence behavior, as has been observed with trapped ions [20]. 
We also attempted to make superstable Hanle-effect MOTs by using rectified dipole forces, which for high laser intensities can exceed the usual MOT scattering forces [21]. With larger trap spring constants and trap temperatures that are comparable to regular MOTs, we expected to realize tighter atom confinement. We found, however, that with such traps the field gradients for which $\tau_{\text {diff }}=1 \mathrm{sec}$ were always substantially lower (typically $\left.B^{\prime}<100 \mathrm{G} / \mathrm{cm}\right)$ than those for a normal $\sigma^{+}-\sigma^{-}$ MOT. This suggests that strong Hanle-type stimulated forces, although effective in $1 \mathrm{D}$, may not be effective in 3D MOTs.

In summary, we examined magneto-optical traps in the tight-confinement limit, and have made the first measurements of the diffusive loss rate as a function of laser intensity and detuning, and MOT field gradient. The data are well modeled at low intensities using a simple phenomenological Fokker-Planck analysis, which supports our hypothesis that atom diffusion is the primary trap loss mechanism in the limit of tight confinement. Extending the model with the addition of an ad hoc high-intensity rolloff, we find a minimum transient MOT size of $\sim 5 \mu \mathrm{m}$, a robust result which is insensitive to the model details. These results apply to generic MOTs, with random (and fluctuating) phases of the different laser beams; further investigation of tight MOTs using phasestable lasers may be quite interesting, potentially leading to controlled submicron atom trapping in a single optical lattice site.

This research was supported in part by funding from Millard and Muriel Jacobs, and the California Institute of Technology.

*Present address: Arroyo Optics, 1646 17th St., Santa Monica, CA 90404.

${ }^{\dagger}$ Electronic address: kgl@ sundog.caltech.edu

[1] E. L. Raab, M. Prentiss, A. Cable, S. Chu, and D.E. Pritchard, Phys. Rev. Lett. 59, 2631 (1987).

[2] T. Walker, D. Sesko, and C. Wieman, Phys. Rev. Lett. 64, 408 (1990); A. M. Steane, M. Chowdhury, and C. J. Foot, J. Opt. Soc. Am. B 9, 2142 (1992); W. Ketterle et al., Phys. Rev. Lett. 70, 2253 (1993); C. G. Townsend et al., Phys. Rev. A 53, 1702 (1996).

[3] D. Sesko et al., Phys. Rev. Lett. 63, 961 (1989); M.H. Anderson et al., Phys. Rev. A 50, R3597 (1994).
[4] A. M. Steane and C. J. Foot, Europhys. Lett. 14, 231 (1991); C. J. Cooper et al., Europhys. Lett. 28, 397 (1994).

[5] M. Drewson et al., Appl. Phys. B 59, 283 (1994).

[6] K. D. Stokes et al., Phys. Rev. Lett. 67, 1997 (1991); J. R. Gardner, M. L. Marable, G. R. Welch, and J.E. Thomas, Phys. Rev. Lett. 70, 3404 (1993); P. Storey, M. Collett, and D. Walls, Phys. Rev. A 47, 405 (1993); S. Kunze, G. Rempe, and M. Wilkens, Europhys. Lett. 27, 115 (1994).

[7] J. Nellessen, J. Werner, and W. Ertmer, Opt. Commun. 78, 300 (1990); E. Riis, D. Weiss, K. Moler, and S. Chu, Phys. Rev. Lett. 64, 1658 (1990).

[8] G. Gwinner et al., Phys. Rev. Lett. 72, 3795 (1994); Z-T. Lu et al., Phys. Rev. Lett. 72, 3791 (1994).

[9] P. A. Willems, Ph.D. thesis, California Institute of Technology, 1997; P. A. Willems, R. A. Boyd, J. L. Bliss, and K. G. Libbrecht (to be published).

[10] Collisional loss is usually parametrized in terms of the decay of the average atom density $\dot{n}=-\beta^{*} n^{2}$, which is a more suitable notation when $N$ is large; these are related by $\beta=\beta^{*} / V$, where $V$ is the effective volume of the trapped atoms. For small $N, \beta$ is independent of $N$.

[11] P. A. Willems and K. G. Libbrecht, Phys. Rev. A 51, 1403 (1995). For the present investigations a new and larger version of this system was used.

[12] Single atoms in MOTs have been observed previously by $\mathrm{Z}$. Hu and H. J. Kimble, Opt. Lett. 19, 1888 (1994); F. Ruschewitz et al., Europhys. Lett. 34, 651 (1996); D. Haubrich et al., Europhys. Lett. 34, 663 (1996).

[13] The collisional decay curve was generated by extrapolating the density from measurements at low $B^{\prime}$, assuming $n \propto N\left(B^{\prime}\right)^{3 / 2}$. At high $B^{\prime}$ the collisional loss rate increases as the trap depth decreases, which explains the observed discrepancy in the two-atom lifetime in Fig. 1 at large $B^{\prime}$. The one-atom lifetime at low $B^{\prime}$ is limited by loss mechanisms other than diffusion.

[14] P. Kohns et al., Europhys. Lett. 22, 517 (1993).

[15] S. Chandrasekhar, Rev. Mod. Phys. 15, 1 (1943).

[16] C. G. Townsend et al., Phys. Rev. A 52, 1423 (1995).

[17] D. Hoffmann, S. Bali, and T. Walker, Phys. Rev. A 54, R1030 (1996).

[18] K. Mølmer, Phys. Rev. A 44, 5820 (1991).

[19] C. D. Wallace et al., J. Opt. Soc. Am. B 11, 703 (1994).

[20] R. G. DeVoe and R. G. Brewer, Phys. Rev. Lett. 76, 2049 (1996).

[21] R. Grimm et al., J. Phys. II (France) 2, 593 (1992); O. Emile et al., Europhys. Lett. 20, 687 (1992). Our trap configuration was the same as the latter reference, with the same polarization precision. 